\title{
IDENTIFICATION OF CHARGE STATES OF INDIUM VACANCIES IN InP
}

\author{
W. Liming, S. Fung and C.D. Beling \\ Department of Physics, University of Hong Kong, Hong Kong, P.R. China
}

The autocorrelation functions of positron-electron pairs in InP with indium vacancies in different charge states are calculated in this work. It is found that the autocorrelation function can be used to identify the charge states of vacancies in solids. In the case of perfect lattice the autocorrelation function oscillates on the lattice with the periodicity of the lattice and decays gradually up to about the third layer of the lattice points. In the case of neutral vacancies, the central peak expands significantly, but the nearest peak and dip are greatly reduced. In the case of negative charge states of vacancies, the central peak, however, contracts slightly compared to the case of the perfect lattice, and the nearest peak and dip and the next-nearest peak nearly disappear.

PACS numbers: 71.15.Mb, 71.15.Pd, 71.55.Eq, 78.70.Bj

The positron annihilation technique has been proved to be a powerful tool to detect the vacancies in solids. This is because positrons can be trapped by vacancies, where the electron density is significantly different from that in the bulk. One may in general obtain different positron lifetimes and momentum distributions of the annihilation radiation in the case of vacancies. In the aspect of theory positron correlation with electrons in solids has been formulated in the local-density approximation (LDA) by Boroński and Nieminen [1]. It results in the correlation potential of the positron in a solid and the enhancement factor of the annihilation rate. This correlation, however, overestimates the annihilation rate, giving rise to slightly smaller positron lifetimes than the experimental data. A density gradient correction (DGC) scheme was proposed by Barbiellini et al. [2] to suppress the overestimation of the LDA. This scheme reproduces positron lifetimes in a great number of solids in good agreement with the experimental data. However, the LDA correlation and the DGC scheme are valid only in bulk states of positrons. In the case of vacancies where positrons may be localized, the positron correlation is poorly known. Therefore, the LDA or DGC correlations are still used in the case of vacancies. It was argued that the positron can be screened by the electrons to become a neutral particle, so that the LDA or DGC correlation is still valid approximately. 
. Recently, Hakala et al. [3] calculated the momentum distributions of annihilation radiation in vacancy clusters in $\mathrm{Si}$. A systematic narrowing of the momentum distributions was found as the size of the clusters increases. In the present work, not the momentum distributions, but the autocorrelation functions (ACF's) are calculated for perfect InP and for InP with indium vacancies in different charge states. The ACF is nothing but the Fourier transform of the momentum distribution. However, the ACF has more structures than the momentum distributions, thus being more suitable to identify vacancies in solids. In the bulk states, the ACF oscillates in space with the lattice periodicity and decays rapidly up to about the third layer of the lattice points. Vacancies in solids can significantly affect the oscillations of the ACF.

The ACF of positron-electron pairs in a solid is defined by

$$
B(\boldsymbol{r})=\sum_{i} \int \Psi_{i}^{\mathrm{ep}}(\boldsymbol{r}) \Psi_{i}^{\mathrm{ep}}(\boldsymbol{r}+\boldsymbol{s}) \mathrm{d} \boldsymbol{s}
$$

where $\Psi_{i}^{\mathrm{ep}}(\boldsymbol{r})$ are the two-particle wave functions of positron-electron pairs. These wave functions can be approximated by the single-particle wave functions of positrons and electrons

$$
\Psi_{i}^{\mathrm{ep}}(r)=\psi_{+}(r) \psi_{i}(r) \sqrt{\gamma(r)}
$$

where $\gamma(r)$ is the enhancement factor, $\psi_{+}(r)$ and $\psi_{i}(r)$ are the wave functions of positrons and valence electrons, respectively.

To model the indium vacancies in InP, a supercell is constructed by enlarging three times the primitive vectors of the fcc primitive cell, thus containing $3 \times 3 \times 3=$ 27 primitive cells and $27 \times 2=54$ atoms (without vacancies). Indium vacancies are created by removing one indium atom from the supercell. Negative charge states of the indium vacancies are formed by adding one or more electrons into the supercell, and a positive charge background is assumed to neutralize the system. The electron and positron wave functions are expanded in plane-wave basis with a cut-off energy of 8 Ry. Norm-conserving pseudopotentials are constructed in the fully separable form of Kleinman-Bylander [4]. The Chadi-Cohen special $k$ point scheme is used to calculate the electron charge density. Ab initio molecular dynamics calculations are performed to determine the electron wave functions and the atomic positions, where only radial relaxation of the vacancies is taken into account. The positron wave function is calculated by directly diagonalizing the positron Hamiltonian. The positron potential includes the point-core potentials of ions, the Hartree potential of electrons, and the correlation potential of Boronski and Nieminen [1]. The DGC of Barbiellini et al. [2] has bren taken into account.

The ACF's of the positron-electron pairs in perfect InP and in InP with indium vacancies in different charge states are calculated according to Eq. (1), as shown in Fig. 1. In the case of perfect InP, the ACF has a central peak, a nearest peak (P1) and dip (D1), a next-nearest peak (P2) and dip (D2), etc. The peaks P1, P2, P3, follow the nodes at 8.16, 13.96, and 24.01 a.u. (atomic units), respectively. These nodes are corresponding to the lattice positions in the [110] direction, but they have been shifted slightly from the exact lattice positions due to the positron correlation. In the case of neutral indium vacancies, the central peak expands significantly, and the following peaks and dips (P1, P2, P3, 


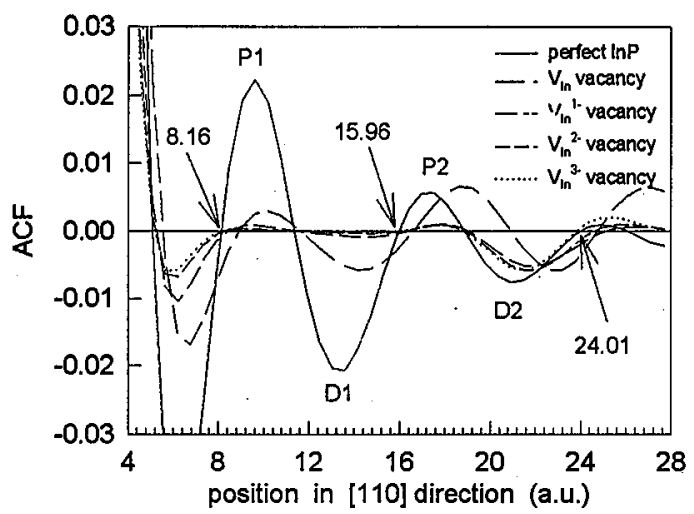

Fig. 1. Autocorrelation functions in the [110] direction in perfect $\operatorname{InP}$ and in $\operatorname{In} \mathrm{P}$ with indium vacancies in different charge states.

$\mathrm{D} 1, \mathrm{D} 2, \ldots)$ all move outwards. This is not surprising because in vacancies the momentum of the positron-electron pairs is reduced. This outward expansion of the ACF corresponds to the narrowing of the momentum distribution spectrum as measured in the two-dimensional angular correlation of .nnihilation radiation (2D-ACAR) or Doppler broadening experiments. It is noted that the atomic relaxation of the neutral indium vacancies is inwards (about :: $i$ ). It was expected that the inward relaxation might cause contraction of the ACF by the authors earlier. This effect, however, has been contradicted by the reduction of momentum of the positron-electron pairs in the neutral vacancies. Nevertheless, this effect does appear in the negative indium vacancies. In this case the central peak contracts, and the following peaks and dips move inwards, corresponding to the inward relaxation of the negative indium vacancies. In addition, the peaks P1 and P2 and the dip D1 nearly disappear in this case. Therefore, one obtains indications of the formation of the neutral and negative indium vacancies in InP. The expansion of the $\mathrm{ACF}$ and the reduction of the $\mathrm{P} 1$ and $\mathrm{D} 1$ indicate the formation of the neutral vacancies, and the disappearance of the $\mathrm{P} 1, \mathrm{P} 2$ and $\mathrm{D} 1$ indicate the formation of the negative indium vacancies. It is concluded that the ACF is suitable to be used to identify the charge states of vacancies in solids.

\section{References}

[1] E. Boroński, R.M. Nieminen, Phys. Rev. B 34, 3820 (1986).

[2] B. Barbiellini, M.J. Puska, T. Korhonen, A. Harju, T. Torsti, R.M. Nieminen, Phys. Rev. B 53, 16201 (1996).

[3] M. Hakala, M.J. Puska, R.M. Nieminen, Phys. Rev. B 57, 7621 (1998).

[4] L. Kleinman, D.M. Bylander, Phys. Rev. Lett. 48, 1425 (1982). 